\title{
Outcome of a combined physiotherapy and podiatry haemophilia clinic: patient perceptions and the effect on ankle bleeds and joint health
}

Charlene Dodd, Alis Trivelli, David Stephensen, Gillian Evans, Miranda Foord

Background: The ankle joint is the most common site of bleeding for people with haemophilia (PWH) in the developed world. Recent surveys suggest that PWH do not always have access to nonsurgical musculoskeletal interventions and that when provided; there is considerable heterogeneity in clinical practice. Aims: To determine patient perceptions and the potential benefits of a new combined multidisciplinary physiotherapy-podiatry haemophilia clinic, and to observe the effect on frequency of bleeds and ankle joint Haemophilia

\section{CHARLENE DODD}

Clinical Specialist Physiotherapist, Haemophilia and Thrombosis Centre, East Kent Hospitals University NHS Foundation Trust, UK. Email: charlene.dodd@anhs.net ALIS TRIVELLI

Clinical Lead Podiatrist for Children, MSK Specialist Podiatrist, Queen Victoria Memorial Hospital, Kent Community Health NHS Foundation Trust, UK

\section{DAVID STEPHENSEN}

Physiotherapist, Haemophilia and Thrombosis Centre, East Kent Hospitals University NHS Foundation Trust, UK

\section{GILLIAN EVANS}

Consultant Haematologist, Director of Kent Haemophilia and Thrombosis Centre, East Kent Hospitals University NHS Foundation Trust, UK

\section{MIRANDA FOORD}

Podiatry Team Lead, Queen Victoria Memorial Hospital, Kent Community Health NHS Foundation Trust, UK

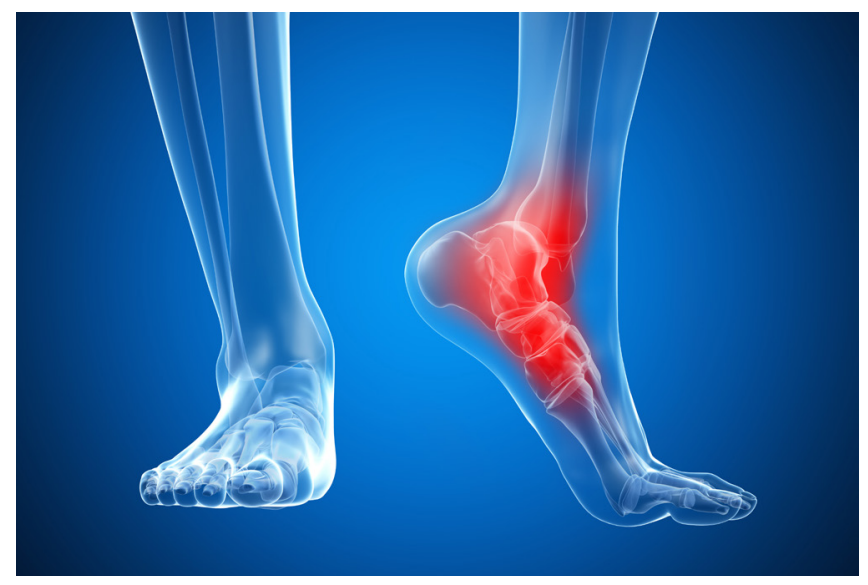

People with haemophilia with a history of ankle bleeds, pain, and foot and/or ankle deformities value the introduction of a combined clinic providing access to a physiotherapist and a podiatrist.

Joint Health Scores (HJHS). Materials and methods: PWH with a history of ankle bleeds, pain, foot and/ or ankle deformities from a single UK haemophilia centre were referred to the clinic from December 2017 to December 2018. Pre- and post-intervention ankle joint HJHS data and ankle annualised joint bleed rate (AJBR) were collected together with a satisfaction questionnaire asking patients their views on the clinic's value, usefulness and their satisfaction after the initial appointment. Results: Twenty-seven PWH (16 children and 11 adults) attended the clinic. All patients agreed or strongly agreed that they

This is an Open Access article distributed under the terms of the Creative Commons Attribution-NonCommercial-NoDerivs License (https://creativecommons.org/licenses/by-nc-nd/3.0/) which permits use and distribution in any medium, provided the original work is properly cited, the use is non-commercial, and no modifications or adaptations are made. Copyright is retained by the authors. 
were satisfied with the new clinic. The combined multidisciplinary nature of the clinic meant that patients only needed to attend one appointment with the expertise of two professionals, rather than attending two separate appointments. All patients reported it "more useful to see the physiotherapist and podiatrist together". There were no statistically significant differences in ankle AJBR or HJHS scores post-intervention compared to pre-intervention. Conclusion: Establishing a multidisciplinary physiotherapy-podiatry clinic for PWH with a history of ankle bleeds, pain, foot and/or ankle deformities appears to increase patient satisfaction. We did not observe a significant change in ankle AJBR or ankle HJHS scores, suggesting they might not be sufficient to evaluate potential benefits to patients.

A larger study incorporating validated tools, focusing on patient-reported foot function, pain, activity and quality of life is needed to confirm if there is any effect of a combined physiotherapy-podiatry intervention on ankle joint AJBR and function.

Keywords: Haemophilia A, Haemophilia B, Physical therapy specialty, Podiatry, Interdisciplinary study, Foot orthoses

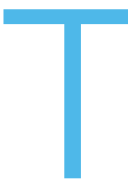

he ankle is the most common site of bleeding in adults and children with haemophilia where there is access to prophylaxis with coagulation factors ${ }^{[1]}$. The long-term consequence of bleeding into joints is arthropathy ${ }^{[2]}$, leading to pain, muscle weakness, atrophy, stiffness, contractures and deformities as well as reduced mobility, balance and proprioception ${ }^{[3]}$. The pathogenesis of haemophilic arthropathy is multifactorial. When a joint bleed occurs, the synovium is responsible for clearing blood from the joint space. However, in the case of ongoing or repeated bleeding the capacity of the synovium is exceeded, leading to iron deposition in the synovium. This induces synovial changes, such as inflammation, hyperplasia and angiogenesis, making the joint more susceptible to renewed bleeding and persistent synovial inflammation (chronic synovitis). Irreversible damage to the articular cartilage occurs due to the direct effect of blood exposure and the secondary effects of inflammatory synovial changes. Blood exposure also has a detrimental impact on the subchondral bone, inducing changes such as cyst formation, epiphyseal enlargement and osteophyte formation. The pathogenic mechanisms resulting in bone changes in haemophilic arthropathy are poorly understood ${ }^{[2]}$.
Ankle arthropathy can involve the tibiotalar and subtalar joints, and the most common associated foot and ankle deformities for people with haemophilia (PWH) include fixed plantarflexion, rearfoot valgus and pes planus foot deformity ${ }^{[4]}$. Brunel et al. (2018) reported that the subtalar joint is less affected than the tibiotalar joint in a small study of adults with haemophilia, and that rearfoot abduction and adduction range of motion during walking was reduced in the presence of subtalar joint arthropathy ${ }^{[5]}$. In a recent study of 163 haemophilia patients, 94 had ankle arthropathy affecting at least one ankle, 56 (59.5\%) of whom presented with a rearfoot malalignment, the most common being a valgus deformity ${ }^{[6]}$.

European and UK guidelines on the care of haemophilia recommend that those with the disorder should have ready access to a range of services provided by a multidisciplinary team of specialists, including availability of a physiotherapy service ${ }^{17,8]}$. However, a 2015 survey of 37 European countries by the European Haemophilia Consortium found that $30 \%$ of countries reported access to physiotherapy for people with haemophilia was only sometimes available ${ }^{\left[{ }^{9]}\right.}$. More recently, a pan-European survey exploring the scope of practice of physiotherapists involved in haemophilia care found considerable heterogeneity in clinical practice ${ }^{[10]}$. The most common physiotherapy interventions reported were muscle strengthening, education, joint range of motion techniques, soft tissue techniques, taping and manual therapy. Use of splinting or orthoses was reported by approximately $25 \%$ of clinicians, although the frequency of application of functional foot orthoses (FFO) is not clear from this survey.

Recent systematic reviews indicate that FFO may be beneficial in reducing pain and forefoot pressures in people with rheumatoid arthritis by redistributing load from painful metatarsal phalangeal joints and controlling subtalar and midtarsal joint forces ${ }^{[11-13]}$. However, there is insufficient evidence to indicate long-term pain relief and reduced disability ${ }^{[13]}$. Several conservative treatments have been described in the management of ankle arthropathy for $\mathrm{PWH}$, including FFO ${ }^{[14-18]}$. Podiatric FFO may be described as functional or accommodative. Functional orthoses apply mechanical forces on joints, creating moments that reduce pressure, shearing or rate of translational forces that would normally be exerted in their absence ${ }^{[19]}$. Accommodative orthoses are primarily used to accommodate or protect an 'at risk' limb, as it is the case with patients suffering from diabetes or osteoarthritis and may also have a secondary 
function of providing mechanical support ${ }^{[19-20]}$. In the vast majority of cases, whether functional or accommodative orthoses are required, there is evidence that prefabricated FFO will be as effective as a bespoke device. Bespoke devices are only prescribed in case of asymmetries between feet or a deformity which a generic orthotic would not be able to accommodate [20].

A small number of studies have reported improvements in pain- and function-related scores for PWH following the provision of FFO [6,18,21]. Existing evidence around ankle bleeds is less clear, with one study reporting a decrease in ankle bleeds ${ }^{[21]}$, and one study reporting a decrease in spontaneous ankle bleeds but an increase in traumatic ankle bleeds following provision of FFO ${ }^{[22]}$. Patients in the latter study reported that they felt safer when wearing the FFO and so increased their participation in sports and activities that could cause sprains and bleeds, which may have accounted for the increase in traumatic bleeding episodes ${ }^{[22]}$. Interestingly, Lobet et al. (2012) described self-reported improvements in pain only in those who reported being satisfied with the FFO ${ }^{[18]}$. There are several limitations to the methodological rigour of these interventional studies including: lack of randomisation or blinding [6,18,21,22]; lack of a comparator group ${ }^{[18,20,22]}$; not all clinically important outcomes were considered ${ }^{[6,21,22]}$; small sample size ${ }^{[18,21]}$; and limited duration of follow-up ${ }^{\text {[21]. }}$

The World Confederation for Physical Therapy defines physical therapy as "services provided by physical therapists to individuals and populations to develop, maintain and restore maximum movement and functional ability throughout the lifespan [...] identifying and maximising quality of life and movement potential within the spheres of promotion, prevention, treatment/intervention, habilitation and rehabilitation."[23] The International Federation of Podiatrists defines podiatry as the "profession of health sciences concerned with the research, prevention, diagnosis and treatment of deformities, pathologies and injuries of the foot and associated structures - in relation with the body as well as the manifestations of systemic diseases - by all appropriate systems and technologies using scientific and professional specialised knowledge."[24]

In 2008, South et al. reported high patient satisfaction of a combined physiotherapy and podiatry clinic for people with haemophilia ${ }^{[25]}$. The rationale for establishing a multidisciplinary physiotherapy-podiatry haemophilia clinic was to combine physiotherapeutic haemophilia musculoskeletal expertise with podiatric foot and ankle musculoskeletal expertise to conservatively manage adults and children with haemophilia with a history of ankle bleeds, pain, foot and/or ankle deformities. The aim of this service evaluation was to establish patient perceptions and the potential benefits of a combined multidisciplinary physiotherapy-podiatry clinic on ankle joint health and ankle annualised bleed rates for people with haemophilia.

Our aim was to observe the effect of a new combined multidisciplinary physiotherapy-podiatry haemophilia clinic on patient perceptions, frequency of ankle joint bleeds and ankle joint Haemophilia Joint Health Scores (HJHS) ${ }^{[26]}$.

\section{MATERIALS AND METHODS}

Prior to December 2017, patients registered at Kent Haemophilia and Thrombosis Centre with a history of ankle bleeds, pain, rearfoot valgus or varus, pes planus or equinus were referred to local community-based podiatry services not linked to the haemophilia treatment centre. However, there was a high non-attendance rate to this clinic. Links were established with the local podiatry service and a combined multidisciplinary physiotherapy-podiatry haemophilia clinic was established within Kent Haemophilia and Thrombosis Centre. At this clinic, patients are assessed concurrently by the clinical specialist physiotherapist in haemophilia (CD) and a musculoskeletal specialist podiatrist (AT), and a treatment plan is discussed and agreed. The clinics are held every two months.

Table 1. General patient characteristics $(n=27)$

\begin{tabular}{|c|c|}
\hline AGE & $\begin{array}{l}\text { MEAN } 23.9 \text { YEARS } \\
\text { (RANGE 4-70 YEARS) } \\
\text { NUMBER OF PATIENTS } \\
\text { (PERCENTAGE) }\end{array}$ \\
\hline \multicolumn{2}{|c|}{ Type of haemophilia } \\
\hline Haemophilia A & $25(92.6 \%)$ \\
\hline Haemophilia B & $2(7.4 \%)$ \\
\hline \multicolumn{2}{|c|}{ Severity of haemophilia } \\
\hline Severe & $17(63.0 \%)$ \\
\hline Moderate & $3(11.1 \%)$ \\
\hline Mild & $7(25.9 \%)$ \\
\hline \multicolumn{2}{|c|}{ Severity of haemophilia } \\
\hline No & $26(96.3 \%)$ \\
\hline Yes & $1(3.7 \%)$ \\
\hline \multicolumn{2}{|c|}{ Severity of haemophilia } \\
\hline Prophylaxis & $17(63.0 \%)$ \\
\hline On-demand & $10(37.0 \%)$ \\
\hline
\end{tabular}


Adults and children with haemophilia registered at Kent Haemophilia and Thrombosis Centre with a history of ankle bleeds, pain, rearfoot valgus or varus, pes planus or equinus who were referred to the combined clinic between December 2017 and December 2018 were provided with a satisfaction questionnaire after the initial appointment. Adults with haemophilia or parents of children with haemophilia who attended the clinic were asked to rate their experience and satisfaction by answering nine questions on a six-point Likert scale, from strongly agree to strongly disagree. Questions focused on communication, care, outcome, convenience, future attendance and recommendation of the service. Pre- and post-intervention ankle joint annualised bleed rate (AJBR) and HJHS data were also collected. This did not include muscle or soft tissue bleeds in the lower leg or foot. Post-intervention data was collected at the patients' subsequent haemophilia clinic review appointments.

Ankle AJBR was collected for six months pre- and post-intervention. For patients on prophylaxis, this was collected retrospectively from Haemtrack, the national system used in the UK for recording haemophilia therapies, accessible by patients and clinicians ${ }^{[27]}$. For patients on on-demand therapy, this data was taken from clinical notes. The HJHS is a clinical outcome measure designed to be carried out by physiotherapists to monitor long-term joint health in people with haemophilia ${ }^{[28]}$. The ankle, knee and elbow joints are assessed, and each joint receives a numeric score out of 20, where 0 represents no clinical signs of arthropathy and 20 represents severe arthropathy. The score can be compared to itself over time to determine if a joint is showing degeneration. Pre- and postintervention HJHS scores for the left and right ankles were combined to give a score out of 40 .

Satisfaction questionnaire data is reported using descriptive statistics. Changes in pre- and postintervention ankle ABR and $\mathrm{HJHS}$ were evaluated with the Wilcoxon signed-rank test in SPSS (version 24) and reported as mean, median and interquartile range. Level of significance was set at $p<0.05$. As data was routinely collected as part of usual care and anonymised, our institution did not require review by an ethical committee. Patients were informed that their anonymised data would be reported and gave their consent for this.

\section{RESULTS}

Twenty-seven ( $n=27)$ PWH (16 children and 11 adults) attended the combined multidisciplinary physiotherapypodiatry haemophilia clinic between December 2017 and December 2018. Table 1 provides an overview of the patients' characteristics. Twenty-six patients (96.3\%) were provided with FFO following their initial appointment which ranged from prefabricated insoles to custom FFO made to particular specifications, including rearfoot and forefoot posting, and heel raises (see Figure 1 for examples). Two patients were lost to follow-up; one patient died, and one patient moved out of area.

\section{Satisfaction questionnaire}

The first 16 patients or carers attending the clinic completed the satisfaction questionnaire. This included ten children, completed by parents/carers, and six adults. All $(n=16)$ agreed or strongly agreed that they were satisfied with the new clinic (see Table 2). All $(n=16)$ agreed or strongly agreed that "it was more useful to see the physiotherapist and podiatrist together", that they "would attend the combined clinic again", and that they "would recommend this service to other people with haemophilia".

\section{Ankle Annualised Joint Bleed Rate (AJBR)}

Ankle AJBR data was available for 24 patients. There was no statistically significant difference in post-intervention ankle AJBR when compared to pre-intervention $(Z=-1.413, p=0.158)$. Mean ankle AJBR was 0.54 preintervention, and 0.25 post-intervention (Table 3 ).

Fourteen patients (58.33\%) had an ankle AJBR of zero pre- and post-FFO provision. Seven patients (29.17\%) had a positive AJBR pre-FFO provision (median 2; IQR 1.5-2.0), which decreased post-FFO provision (median 0; IQR 0.0-0.5). Three patients (12.50\%) had an AJBR of zero pre-FFO provision (median 0.0, IQR 0.0-0.0) but experienced a single traumatic ankle bleed (2 patients) or two traumatic ankle bleeds (1 patient) post-FFO provision (median 1.0, IQR 0.0-1.5).

\section{Ankle Haemophilia Joint Health Score (HJHS)}

Full pre- and post-intervention HJHS data was available for 22 patients. There was no statistically significant difference in the post-intervention ankle joint HJHS when compared to the pre-intervention score $(Z=-$ $0.121, p=0.904)$. Median ankle joint HJHS was 1.0 (IQR $0.0-4.0$ ) pre-intervention, and 0.0 (IQR 0.0-2.25) postintervention (Table 3).

Thirteen patients (59.09\%) had the same HJHS ankle scores pre- and post-FFO provision, of which ten had a score of zero. Four patients $(18.18 \%)$ had a decrease in their HJHS ankle scores post-FFO provision: two patients had a decrease of one point; one had a 
Table 2. Satisfaction questionnaire results

NUMBER OF PATIENTS (PERCENTAGE)

\begin{tabular}{|c|c|c|c|c|c|c|}
\hline Total responses to questionnaire & $16(57.1 \%)$ & & & & & \\
\hline QUESTIONS & SA & A & U & D & SD & N/A \\
\hline $\begin{array}{l}\text { The physiotherapist explained the reason for the } \\
\text { appointment to me prior to my/my child's appointment }\end{array}$ & $15(93.75 \%)$ & $1(6.25 \%)$ & & & & \\
\hline $\begin{array}{l}\text { The physiotherapist and podiatrist explained things in a } \\
\text { way that was easy to understand during the appointment }\end{array}$ & $15(93.75 \%)$ & $1(6.25 \%)$ & & & & \\
\hline $\begin{array}{l}\text { The physiotherapist and podiatrist listened to me } \\
\text { carefully during the appointment }\end{array}$ & $16(100 \%)$ & & & & & \\
\hline $\begin{array}{l}\text { I am satisfied with the care I/my child received during the } \\
\text { appointment }\end{array}$ & $14(87.5 \%)$ & $2(12.5 \%)$ & & & & \\
\hline $\begin{array}{l}\text { I am satisfied with the outcome of the appointment for } \\
\text { me/my child }\end{array}$ & $12(75 \%)$ & $4(25 \%)$ & & & & \\
\hline $\begin{array}{l}\text { I found it more useful to see the podiatrist and } \\
\text { physiotherapist together }\end{array}$ & $12(75 \%)$ & $4(25 \%)$ & & & & \\
\hline $\begin{array}{l}\text { Attending a podiatry appointment at the Haemophilia } \\
\text { Centre is more convenient for me than attending my } \\
\text { local podiatry clinic }\end{array}$ & $14(87.5 \%)$ & $2(12.5 \%)$ & & & & \\
\hline $\begin{array}{l}\text { I would attend the combined physiotherapy/ podiatry } \\
\text { clinic again in the future }\end{array}$ & $14(87.5 \%)$ & $2(12.5 \%)$ & & & & \\
\hline $\begin{array}{l}\text { I would recommend this service to other people with } \\
\text { haemophilia }\end{array}$ & $16(100 \%)$ & & & & & \\
\hline
\end{tabular}

SA: Strongly agree

A: Agree

D: Disagree

U: Undecided SD: Strongly disagree

N/A: Not applicable

Table 3. Ankle HJHS scores and AJBR results

\begin{tabular}{|c|c|c|c|c|}
\hline & MEAN (+SD) & MEDIAN (IQR) & MINIMUM & MAXIMUM \\
\hline HJHS pre-intervention & $2.86(+5.51)$ & $1.0(0.0-4.0)$ & 0.0 & 20.0 \\
\hline HJHS post-intervention & $2.86(+6.15)$ & $0.0(0.0-2.25)$ & 0.0 & 21.0 \\
\hline AJBR pre-intervention & $0.54(+0.93)$ & $0.0(0.0-1.0)$ & 0.0 & 3.0 \\
\hline AJBR post-intervention & $0.25(+0.53)$ & $0.0(0.0-0.0)$ & 0.0 & 2.0 \\
\hline
\end{tabular}

SD: Standard deviation

IQR: Interquartile range

decrease of two points; one had a decrease of four points. Five patients (22.73\%) had an increase in their HJHS ankle scores post-FFO provision: three patients had an increase of one point; one had an increase of two points; and one had an increase of three points. Items of the HJHS that increased were loss of range of motion $(n=3)$, strength $(n=2)$, pain $(n=2)$, and persistence of swelling $(n=1)$. Items of the HJHS that decreased were loss of range of motion $(n=2)$, swelling $(n=1)$, and crepitus $(n=1)$.

\section{DISCUSSION}

We hypothesised that the introduction of a combined multidisciplinary physiotherapy-podiatry clinic would be acceptable to patients, might reduce the frequency of ankle joint bleeds, and might prevent deterioration in ankle joint HJHS. We found high patient satisfaction with the combined clinic approach and no significant effect on ankle AJBR or HJHS.

\section{Patient satisfaction}

Patients reported high levels of satisfaction with a combined multidisciplinary physiotherapy-podiatry haemophilia clinic. The combined multidisciplinary nature of the clinic meant that patients only needed to attend one appointment with the expertise of two professionals together at the same time, rather than attending two separate appointments. All patients reported it "more useful to see the physiotherapist and podiatrist together". As the clinic took place in the 
Haemophilia and Thrombosis Centre, it was also held in a familiar setting for the patients. All patients said they "would attend the clinic again" and "would recommend the service to other people with haemophilia". No further questionnaires were distributed after the first 16 patients as no new information was being found from the questionnaire results.

Rome et al. (2013) also reported high levels of satisfaction with a new podiatric rheumatology service ${ }^{[28]}$. Although this was not a combined clinic, $84 \%$ of patients reported they were very satisfied with the service and $63 \%$ reported they found the podiatry service very useful.

Patient satisfaction may be positively associated with adherence to care. This has been demonstrated in other long-term health conditions. In a questionnairebased study of patients with psoriatic arthritis, Leung et al. (2009) reported a positive correlation between level of involvement in healthcare and satisfaction $(p<0.001)$ and those with high levels of involvement reported better physical and mental health ${ }^{[29]}$. In a survey-based study of patients with diabetes, Albright et al. (2001) reported that patient satisfaction was significantly associated with self-care behaviours including: taking medication ( $p=0.003)$; following a diabetic diet $(p=0.000)$; and glucose self-monitoring $(p=0.016)^{[30]}$. In a cross-sectional study of adults receiving HIV care, Dang et al. (2013) found patients who reported "excellent" adherence to their antiretroviral therapy were significantly more satisfied with their HIV care than those patients who were not adherent $(p<0.0001)^{[31]}$.

Impact of functional foot orthoses on ankle AJBR We did not observe a significant change in ankle AJBR following provision of an in-shoe FFO. It was interesting, however, that at six months' follow-up, $87.5 \%$ of patients maintained an AJBR of zero or had a lower AJBR post-FFO intervention, suggesting that the combined clinic is a safe and acceptable option.

Figure 1. Examples of functional foot orthoses provided to patients

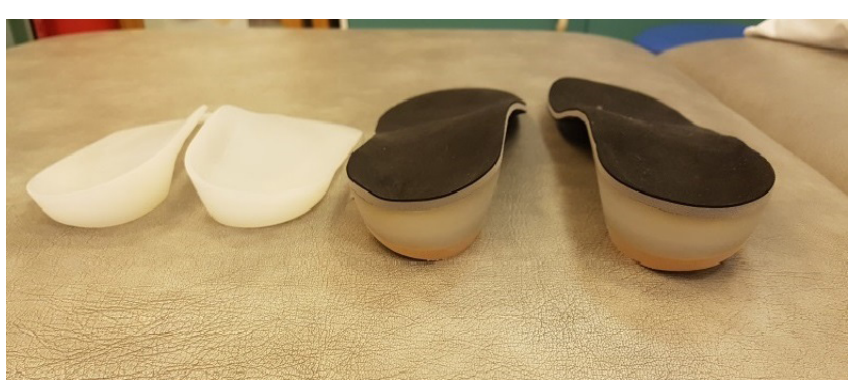

Previous studies have reported a decrease in ankle bleeds following the provision of FFO. Slattery and Tinley (2001) reported a decrease in ankle bleeds for all 16 subjects supplied with FFO at six-week follow-up ${ }^{[21]}$. Filho et al. (2006) reported a significant decrease in spontaneous ankle bleeding episodes for 43 haemophilia patients in the six months following provision of insoles +/- Air Stirrup brace compared to the six months before provision [22]; however, they also reported a significant increase in traumatic ankle bleeding episodes. Patients in this study reported that they felt safer when wearing the insoles +/- Air Stirrup brace, and so increased their participation in sports and activities that could cause sprains and bleeds, which may have accounted for the increase in traumatic bleeding episodes ${ }^{[22]}$.

\section{Impact of functional foot orthoses on ankle HJHS} To date, no previous studies have examined the effect of FFO on HJHS scores in people with haemophilia. We did not observe a significant change in ankle HJHS following provision of an in-shoe FFO. For most patients $(77.27 \%)$ there was no change in ankle $\mathrm{HJHS}$ scores post-FFO intervention. This suggests that the summed joint HJHS might not be sufficient to evaluate the potential benefits to patients of this type of intervention. Tools focusing on patient-reported foot function, including pain, activity and quality of life, together with quantitative evaluation of physical function might be more appropriate.

When considering individual items of the HJHS, there was considerable inter-patient variability when comparing pre- and post-intervention scores. This may be due to the fact that changes in individual items of the $\mathrm{HJHS}$ are challenging to interpret due to the nature of unequal categories in the ordinal scoring criteria and the ceiling effects of the loss of range of motion item.

$\mathrm{HJHS}$ items that increased included loss of range of motion, strength, pain, and persistence of swelling. Two patients had activity-related ankle bleeds, which may account for an increase in pain, loss of range of motion and a decrease in strength. One patient developed chronic synovitis in the left ankle, which resulted in persistent swelling, despite a decrease in ankle AJBR post-intervention (one bleed) compared to pre-intervention (three bleeds). Two patients had no bleeds but had an increase in loss of range of motion. Both patients had established ankle arthropathy in one or both ankles and had shown a trend for decreasing range of motion over several $\mathrm{HJHS}$ scores prior to the intervention. 
HJHS items that decreased included swelling, loss of range of motion, and crepitus. One patient had a decrease in swelling, which could be attributed to having no bleeds post- intervention compared to two bleeds pre-intervention. Two patients had an increase in range of motion and one patient had a decrease in crepitus post-intervention. All three patients had no bleeds or injuries pre- or post-intervention and it is therefore unclear why these items changed.

\section{Study limitations}

There are several limitations to our study. Ankle AJBR data were only collected for six months pre- and post-intervention. In addition, it is difficult to use AJBR as an outcome measure in a mixed cohort of adults and children.

The satisfaction questionnaire used was not validated and we did not include a patient-reported functional outcome measure, a pain-specific outcome measure, or outcomes such as delayed requirements for surgery, changes in analgesia use, walking distance, recovery time following activity, and early morning stiffness. It may have also been useful to include gait analysis and abnormalities among the clinic referral criteria alongside structural abnormalities.

A large number of ankle HJHS scores for our patients were low, with $45.45 \%$ patients having a score of zero pre- and post-intervention. The HJHS may therefore not have been the most appropriate outcome measure to use. In addition, use of the HJHS over a 12-month period may not be sensitive enough on its own to show change, improvements or deterioration.

The clinic physiotherapist was not blinded to patients' participation in the clinic, introducing potential assessor bias in the HJHS examination and scoring. In addition, although we have not addressed the limitations of the other studies in the current paper, we have found that currently recommended outcomes (AJBR and HJHS) may not be sufficient to evaluate potential patient benefits.

\section{CONCLUSION}

This service evaluation highlights the potential benefits of a combined physiotherapy-podiatry multidisciplinary clinic approach rather than attendance at separate clinics for PWH with a history of ankle bleeding, pain, foot and/or ankle deformities. Patient satisfaction with our clinic was high, and the clinic's success has led to an increase in clinic frequency.

We did not observe a significant change in ankle AJBR; however, most patients $(87.5 \%)$ continued to have an ankle AJBR of zero or a lower AJBR post-FFO intervention. We did not observe a significant change in ankle HJHS scores; however, scores did not show deterioration for most patients (77.27\%), indicating that this type of intervention is safe for PWH. Given the small sample size, we are unable to draw firm conclusions about ankle AJBR and HJHS scores post-FFO intervention. A larger study incorporating validated tools focusing on patient-reported foot function, pain, activity and quality of life, together with quantitative evaluation of physical function, is needed to confirm whether there is any effect of a combined physiotherapy-podiatry intervention on ankle AJBR and function.

\section{ACKNOWLEDGEMENTS}

CD and AT developed the clinic and collected data.

CD, AT and DS contributed to data analysis and interpretation. CD, AT, DS, GE and MF drafted and approved the final version of the manuscript.

The authors have advised no interests that might be perceived as posing a conflict or bias.

Informed consent has been obtained from the participants in this study.

\section{ORCID}

Charlene Dodd (iD https://orcid.org/0000-0002-0416-5916 Alis Trivelli (iD https://orcid.org/0000-0002-3455-5587 David Stephenson (iD https://orcid.org/0000-0002-6175-3343

\section{REFERENCES}

1. Stephensen D, Tait R, Brodie N, et al. Changing patterns of bleeding in patients with severe haemophilia A. Haemophilia 2009; 15(6): 1210-14. doi: 10.1111/j.1365-2516.2008.01876.x.

2. Roosendaal G, Lafeber F. Pathogenesis of haemophilic arthropathy. Haemophilia 2006; 12(3): 117-21.

3. Mulder K, Katzerova M. Chronic sequels: Haemophilic arthropathy and exercise. In: Kleijn P, Mauser-Bunschoten E (ed.). Physiotherapy Management in Haemophilia. The Netherlands: HH Global; 2017.

4. Pasta G, Forsyth A, Merchan C, et al. Orthopaedic management of haemophilia arthropathy of the ankle. Haemophilia 2008; 14(3): 170-6. doi: 10.1111/j.13652516.2008.01720.x.

5. Brunel T, Lobet S, Deschamps K, et al. Reliability and clinical features associated with the IPSG MRI tibiotalar and subtalar joint scores in children, adolescents and young adults with haemophilia. Haemophilia 2018; 24(1): 141-48. doi: 10.1111/ hae.13368

6. De la Corte-Rodriguez H, Rodriguez-Merchan E, AlvarezRoman M, Martin-Salces M, Jimenez-Yuste V. Hindfoot malalignment in adults with haemophilic ankle arthropathy: The importance of early detection and orthotic treatment. Haemophilia 2019; 25(3): 500-508. doi: 10.1111/hae.13680. 
7. Colvin BT, Astermark J, Fischer K, et al.; Interdisciplinary Working Group. European principles of haemophilia care. Haemophilia 2008; 14(2): 361-74. doi: 10.1111/j.13652516.2007.01625.x.

8. NHS England. 2013/14 NHS Standard Contract for Haemophilia (All Ages). Section B Part 1 - Service Specifications. B05/S/a. Available from https://www.england. nhs.uk/wp-content/uploads/2013/06/b05-haemophilia.pdf (accessed 8 April 2020).

9. O'Mahony B, Savani L, O'Hara J, Bok A. Haemophilia care in Europe - a survey of 37 countries. Haemophilia 2017; 23(4): e259-e266. doi: 10.1111/hae.13263.

10. Stephensen D, de Kleijn P, Matlary R, et al. Scope of practice of haemophilia physiotherapists: a European survey. Haemophilia 2019; 25(3): 514-20. doi: 10.1111/hae.13727.

11. Conceição CSD, Gomes Neto M, Mendes SM, Sá KN, Baptista AF. Systematic review and meta-analysis of effects of foot orthoses on pain and disability in rheumatoid arthritis patients. Disabil Rehabil 2015; 37(14): 1209-13. doi: 10.3109/09638288.2014.961654.

12. Hennessy K, Woodburn J, Steultjens MP. Custom foot orthoses for rheumatoid arthritis: a systematic review. Arthritis Care Res (Hoboken) 2012; 64(3): 311-20. doi: 10.1002/ acr.21559.

13. Gijon-Nogueron G, Ramos-Petersen L, Ortega-Avila AB, Morales-Asencio JM, Garcia-Mayor S. Effectiveness of foot orthoses in patients with rheumatoid arthritis related to disability and pain: a systematic review and meta-analysis. Qual Life Res 2018; 27(12): 3059-69. doi: 10.1007/s11136018-1913-5.

14. Heijnen L, Roosendaal G, Heim M. Orthotics and rehabilitation for chronic hemophilic synovitis of the ankle. An overview. Clin Orthop Relat Res 1997; 343: 68-73.

15. Battistella L. Maintenance of musculoskeletal function in people with haemophilia. Haemophilia 1998; 4(2): 26-32.

16. Ribbans W, Rees J. Management of equinus contractures of the ankle in haemophilia. Haemophilia 1999; 5(1): 46-52.

17. Heijnen L, Heim M, In Der Maur H; World Federation of Hemophilia. Hemophilia. Manufactured shoes and orthopaedic shoes. Haemophilia 2000; 6(1): 4-6.

18. Lobet S, Detrembleur C, Lantin A, Haenecour L, Hermans C. Functional impact of custom-made foot orthoses in patients with haemophilic ankle arthropathy. Haemophilia 2012; 18(3): e227-35. doi: 10.1111/j.1365-2516.2011.02711.x.

19. Hawke F, Burns J. Understanding the nature and mechanism of foot pain. J Foot Ankle Res 2009; 2: 1. doi: 10.1186/17571146-2-1.

20. Gallagher KS, Godwin J, Hendry GJ, Steultjens M, Woodburn J. A protocol for a randomised controlled trial of prefabricated versus customised foot orthoses for people with rheumatoid arthritis: the FOCOS RA trial [Foot Orthoses - Customised v
Off-the-Shelf in Rheumatoid Arthritis]. J Foot Ankle Res 2018; 31: 11-24. doi: 10.1186/s13047-018-0272-3.

21. Slattery $M$, Tinley P. The efficacy of functional foot orthoses in the control of pain in ankle joint disintegration in hemophilia. J Am Podiatr Med Assoc 2001; 91(5): 240-4.

22. Jorge Filho $D$, Battistella L, Lourenço $C$. Computerized pedobarography in the characterization of ankle-foot instabilities of haemophilic patients. Haemophilia 2006; 12(2): 140-6.

23. World Confederation for Physical Therapy. Policy statement: Description of physical therapy. 2017; Available from https:// www.wcpt.org/policy/ps-descriptionPT (accessed 8 April 2020).

24. International Federation of Podiatrists. What is Podiatry. 2018. Available from https://www.fip-ifp.org/what-we-do/what-ispodiatry/ (accessed 8 April 2020).

25. South A, Short L, Richards M. Utility of a combined physiotherapy and podiatry clinic for adults and children with haemophilia. SessionB-27: Musculoskeletal free papers 02 / 12 FP 31. Haemophilia 2008; 14 Suppl 2: 1-157 (76). doi: 10.1111/j.1365-2516.2008.01725.x.

26. Feldman B, Funk S, Bergstrom B, et al. Validation of a new pediatric joint scoring system from the International Hemophilia Prophylaxis Study Group: validity of the hemophilia joint health score. Arthritis Care Res (Hoboken) 2011; 63(2): 223-30. doi: 10.1002/acr.20353.

27. Haemtrack. What is Haemtrack? Available from http:// haemtrack.mdsas.com/\#about (accessed 8 April 2020).

28. Rome K, Erikson K, Ng A, Gow P, Sahid H, Williams A. A new podiatry service for patients with arthritis. N Z Med J 2013; 126 (1370): 70-77.

29. Leung YY, Tam LS, Lee KW, Leung MH, Kun EW, Li EK. Involvement, satisfaction and unmet health care needs in patients with psoriatic arthritis. Rheumatology (Oxford) 2009; 48(1): 53-56. doi: 10.1093/rheumatology/ken410.

30. Albright TL, Parchman M, Burge SK; RRNeST Investigators. Predictors of self-care behavior in adults with type 2 diabetes: an RRNeST study. Fam Med 2001; 33(5): 354-60.

31. Dang B, Westbrook R, Black W, Rodriguez-Barradas M, Giordano T. Examining the link between patient satisfaction and adherence to HIV care: a structural equation model. PLOS ONE 2013; 8(1): e54729. doi: 10.1371/journal.pone.0054729.

HOW TO CITE THIS ARTICLE:

Dodd C, Trivelli A, Stephensen D, Evans G, Foord M.

Outcome of a combined physiotherapy and podiatry

haemophilia clinic: patient perceptions and the effect on

ankle bleeds and joint health. J Haem Pract 2020; 7(1):

37-44. https://doi.org/10.17225/jhp00153.

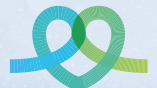

The Journal of Haemophilia Practice
An open-access journal for sharing

experience in the care of people with bleeding disorders 